This item was submitted to Loughborough's Research Repository by the author.

Items in Figshare are protected by copyright, with all rights reserved, unless otherwise indicated.

\title{
Toward a mechanistic understanding of microfluidic droplet-based extraction and separation of lanthanides
}

PLEASE CITE THE PUBLISHED VERSION

https://doi.org/10.1016/j.cej.2018.09.043

\section{PUBLISHER}

(C) Elsevier

VERSION

AM (Accepted Manuscript)

\section{PUBLISHER STATEMENT}

This paper was accepted for publication in the journal Chemical Engineering Journal and the definitive published version is available at https://doi.org/10.1016/j.cej.2018.09.043.

LICENCE

CC BY-NC-ND 4.0

\section{REPOSITORY RECORD}

Zhang, Hao, Huizhi Wang, Xiaojiao Luo, Dennis Y. Leung, Qishou Pang, Hong Xu, Li Zhang, and Jin Xuan. 2018. "Toward a Mechanistic Understanding of Microfluidic Droplet-based Extraction and Separation of Lanthanides". figshare. https://hdl.handle.net/2134/35224. 
Toward a mechanistic understanding of microfluidic droplet-based extraction and separation of lanthanides

Hao Zhang ${ }^{\mathrm{a},{ }^{*}}$, Huizhi Wang ${ }^{\mathrm{b}}$, Xiaojiao Luo ${ }^{\mathrm{c}}$, Dennis YC Leung ${ }^{\mathrm{d}}$, Qishou Pang ${ }^{\mathrm{a}}$, Hong

$$
\mathrm{Xu}^{\mathrm{e}} \text {, Li Zhang }{ }^{\mathrm{e}} \text {, Jin Xuan }{ }^{\mathrm{f}}
$$

${ }^{a}$ School of Mechanical and Electrical Engineering, Jiangxi University of Science and Technology, Ganzhou, China

${ }^{\mathrm{b}}$ Department of Mechanical Engineering, Imperial College London, Exhibition Road, South Kensington Campus, London, United Kingdom

${ }^{\mathrm{c}}$ School of Engineering \& Physical Sciences, Heriot-Watt University, Edinburgh,

$$
\text { United Kingdom }
$$

${ }^{\mathrm{d}}$ Department of Mechanical Engineering, The University of Hong Kong, Pokfulam

$$
\text { Road, Hong Kong }
$$

${ }^{\mathrm{e}}$ State Key Laboratory of Chemical Engineering, School of Mechanical and Power Engineering, East China University of Science and Technology, Shanghai, China

${ }^{\mathrm{f}}$ Department of Chemical Engineering, Loughborough University, Loughborough, United Kingdom

* Corresponding Author, Tel: +86 (0797) 8312137; Fax: +86 (0797) 8312137; Email address: haozhang@jxust.edu.cn 


\begin{abstract}
Droplet-based microfluidic extraction is a promising way for effective lanthanides extraction due to its outstanding mass transfer performance. The separation process can be greatly enhanced with the droplet-based microfluidic extraction technique. However, the interactions between mass transfer, microfluidic dynamics and extraction kinetics are still unclear, which has hindered further manipulation on microfluidic extraction to boost extraction performance. In this study, the mechanisms of microfluidic droplet-based extraction and separation intensification of lanthanides are for the first time unveiled by using a numerical simulation model. The limiting factors for the performance of droplet-based microfluidic extraction are identified through a model-based parametric analysis. The numerical analyses provide a comprehensive understanding of droplet-based microfluidic extraction systems and offer operation and optimization guidelines for future research in this area.
\end{abstract}

Key words: Droplet; Microfluidic; Extraction; Separation; Lanthanide 


\section{Nomenclature:}

$A \quad$ area of the interface $\left(\mathrm{m}^{2}\right)$

c concentration of species $\left(\mathrm{mol} \mathrm{L}^{-3}\right)$

d diameter of microchannel (m)

$D$ distribution ratio

$\bar{D}$ diffusivity $\left(\mathrm{m}^{2} \mathrm{~s}^{-1}\right)$

$E \quad$ extraction percentage

$k_{f}$ forward rate constants $\left(\mathrm{s}^{-1}\right)$

$k_{r} \quad$ reverse rate constants $\left(\mathrm{s}^{-1}\right)$

$k_{m}$ total mass transfer rate $\left(\mathrm{s}^{-1}\right)$

$L \quad$ length of microchannel (m)

$L_{s} \quad$ length of slug (m)

$n \quad$ reaction order

$p$ pressure $(\mathrm{Pa})$

$r \quad$ the radius of the droplet contact surface (m)

$R$ generation or consumption rate of species $\left(\mathrm{mol} \mathrm{m}^{-3} \mathrm{~s}^{-1}\right)$

$\overline{t_{m}}$ time scale of total mass transport toward interface (s)

$\bar{t}_{c}$ time scale of convection transport in flow direction (s)

$u \quad$ vecolity $\left(\mathrm{m} \mathrm{s}^{-1}\right)$

$V \quad$ volume $\left(\mathrm{m}^{3}\right)$

Inf intensification factor $\alpha \quad$ contact angle (degree)

$\beta$ separation factor

$\rho \quad$ density $\left(\mathrm{kg} \mathrm{m}^{-3}\right)$

$\tau \quad$ the viscosity stress tensor $(\mathrm{Pa})$

Subscript

$i \quad$ specie

$a q$ aqueous

org organic

gen generation

con consumption 


\section{Introduction}

Lanthanide is a series of elements with astounding physicochemical properties, which has long attracted lots of research interests in material science areas, such as magnetic, optical and luminescent materials, as well as catalysts et al.[1,2]. Solvent extraction is the most widely applied technology for lanthanides separation and purification, which is a very important process for rare earths metallurgy. Mixer-settler is a commonly utilized lanthanides separation device which consists of a mixing chamber bringing convective mixing of immiscible phases and a settling chamber performing phase separation[3]. However, due to the chemical resemblance of lanthanides, it is difficult to achieve their separation thus cascade separation operation with thousands of mixer-settler has to be implemented[1]. Integration of microfluidics into solvent extraction can introduce many benefits for improving interface mass transport, such as shortening the mass transport path and enlarging surface-to-volume ratio[4, 5].

Microfluidic extraction can be categorized into two types according to flow pattern, i.e., co-laminar microfluidic extraction and droplet-based microfluidic extraction. Experiments on both configurations have proved that the microfluidics is a highly efficient approach to achieve extraction equilibrium in much shorter time compared with traditional bulk devices[6-10]. In a co-laminar microfluidic extraction system, since aqueous and organic streams flow side by side, it is convenient to achieve phase separation at the outlet. In order to maintain the co-laminar flow pattern for two immiscible streams, high capillary number $(\mathrm{Ca})$ is required which associates 
with a flow velocity of $40-400 \mathrm{~mm} \mathrm{~s}^{-1}$ according to the shape of microchannel and fluid property[11]. High Ca operation condition leads to shortening of residence time in the microchannel. Therefore, co-laminar microfluidic extraction is suitable for extraction reactions with fast kinetics. In a droplet-based microfluidic extraction system, benefiting from the vortexes induced by shear stress, the mass transfer rate can be ten to thousand times higher than the one in traditional extraction system[12-15]. Since the operation flow velocity of droplet-based flow pattern keeps in a relatively low range (less than $200 \mathrm{~mm} \mathrm{~s}^{-1}$ [11]), this configuration is suitable for extraction reactions with slow kinetics.

In addition to the excellent mass transfer performance, it has also been proved that separation intensification can be achieved in a microfluidic extraction system, which is a crucial advantage for lanthanides separation[16, 17]. Separation performance of microfluidic extraction was found double as the one in batch reactor[17]. Although the initial data show us the notable separation performance of microfluidic extraction, the interactions among mass transfer, microfluidic dynamics and extraction kinetics are still unknown. To the best of our knowledge, no work has been reported to give a mechanistic understanding of the separation intensification in a microfluidic extraction system, which is vital for manipulating microfluidic extraction to further boost on separation performance.

This paper presents a numerical study on a droplet-based microfluidic extraction system to explore the mass transfer characteristics of extraction and mechanism of separation intensification in microfluidic extraction. The extraction processes of $\mathrm{La}^{3+}$ 
and $\mathrm{Eu}^{3+}$ were chosen in the model, because the extraction kinetics of $\mathrm{La}^{3+}$ and $\mathrm{Eu}^{3+}$ are well documented and entirely different[18]. Lanthanum has bright application prospects for catalysis, superconducting materials, oxide electrolyte etc. Lanthanum-based perovskite oxides demonstrate promising catalytic performance as for electrochemical conversion, heterogeneous oxidation reaction, thermochemical water and carbon dioxide splitting[19, 20]. Lanthanum hydrides have been considered a brilliant high-temperature superconducting material at accessible pressure[21]. Lanthanum-based solid oxide exhibits fast oxide-ion conducting properties, which makes it a favorable electrolyte for solid oxide fuel cells[22, 23]. Europium-based phosphorescent materials show outstanding luminescent properties including high photostability, extremely narrow emission lines, long lifetime, and tunable wavelength from blue to red, which makes them essential in display, screen and bioprobe applications[24, 25]. The performance limiting factors of lanthanide extraction in microchannel were clarified. It is found that separation performance of lanthanides in a droplet-based microfluidic extraction system depends on the distinction of extraction kinetic rate of lanthanides, and can be manipulated through adjusting the flow velocity.

\section{Numerical model}

\subsection{Problem statement and computational geometry}

Cylinder-shaped slug geometry was built for the microfluidic extraction system as shown in Fig. 1. Since the slugs are distributed periodically and centrosymmetric, 
only a quarter of one droplet unit was modeled. The diameter and the length of geometry are $1 \mathrm{~mm}$ and $2.67 \mathrm{~mm}$, respectively. The length of droplet is $1.48 \mathrm{~mm}$. The shape of interfacial surface can be defined from the following equations[26]:

$$
\begin{gathered}
y=\sqrt{r_{1}^{2}-x^{2}}+\sqrt{r_{2}^{2}-z^{2}} \\
\int_{0}^{d / 2} \int_{0}^{d / 2} y d x d z=\frac{V}{8} \\
\left.\frac{\partial y}{\partial z}\right|_{z=\frac{d}{2}}=-\tan (\alpha) \\
\left.\frac{\partial y}{\partial x}\right|_{x=\frac{d}{2}}=-\tan (\alpha)
\end{gathered}
$$

where $x, y, z$ denotes the coordinate, $V$ is the volume of the slug, $r_{l}$ and $r_{2}$ denotes the radius of the droplet contact surface in the $x$ and $z$ direction, respectively; $\alpha$ is the contact angel of the droplet at the wall, $d$ is the width of channel.

The lanthanide ions i.e. $\mathrm{La}^{3+}$ and $\mathrm{Eu}^{3+}$ in droplet were extracted into organic solvent through Trivalent Actinide-Lanthanide Separation by Phosphorus reagent Extraction from Aqueous Komplexes (TALSPEAK) process[18].

\subsection{Governing equations}

The hydrodynamics of droplet-based flow in microchannel are governed by continuity of mass and conservation of momentum, which were treated as stationary[27, 28]:

$$
\begin{gathered}
\nabla \cdot(\rho u)=0 \\
\nabla \cdot(\rho u u)=-\nabla \cdot p+\nabla \cdot \tau
\end{gathered}
$$

in which, $\rho$ refers to the density of the fluid, $u$ denotes the velocity vector, $p$ is the 
pressure, $\tau$ is the viscous stress vector.

The mass transfer of metal ions and lanthanide ligand complex involved in microfluidic extraction is determined by conservation equation of species:

$$
\frac{\partial c_{i}}{\partial t}+\nabla \cdot\left(-\overline{D_{i}} \nabla c_{i}\right)+u \cdot \nabla c_{i}=R_{i}
$$

where $c$ is the concentration of species, $\bar{D}$ denotes diffusion coefficient. Since metal ions transport from aqueous solution to organic solution generating lanthanide ligand complex, $R$ stands for the generation rate of extraction product, i.e. lanthanide complexes, and consumption rate of species, i.e. metal ions. The equation for conservation of species is treated as time dependent, because the concentration profiles in microchannel evolves along with time.

The extraction process of lanthanide from aqueous solution (lanthanide ions) to organic solvent (lanthanide ligand complex) can be considered as a first order equilibrium reaction with the forward and reverse rate constants $k_{f}$ and $k_{r}$ when the extractant is excessive[18]:

$$
c_{a q} \longleftrightarrow c_{o r g}
$$

In the result, the net consumption rate of lanthanide ions in aqueous solution, as well as the net generation rate of lanthanide ligand complexes in organic solvent, can be given as follow:

$$
R_{\text {gen }, i}=-R_{\text {con }, i}=k_{f, i} c_{a q, i}-k_{r, i} c_{\text {org }, i}
$$

\subsection{Boundary conditions}

The wall condition for slug geometry was set moving wall[26]: 


$$
u_{\text {wall }}=u
$$

At the interfacial surfaces between aqueous phase and solvent phase, the velocities of both phases are equalized to satisfy the momentum continuity. Therefore, the flow continuity condition was set on interfacial surfaces:

$$
u_{a q}=u_{\text {org }}
$$

However, since there is no momentum transfer at the interfacial surface in the direction perpendicular to the surface, the perpendicular velocity at the surface was set 0 .

$$
u \cdot \vec{n}=0
$$

Periodic conditions were set on both cross section surfaces.

$$
u_{1}=u_{2}=u
$$

In order to solve the mass transfer equations (Eq. 7), initial concentration of every species were given in each phase.

$$
c_{i, t=0 s}=c_{i, 0}
$$

Periodic concentration conditions were set on both cross section surfaces.

$$
c_{1}=c_{2}
$$

Symmetry conditions were set on the symmetric surfaces. A thin extraction reaction layer was modeled at the interfacial surface to accomplish extraction reactions.

\subsection{Numerical solution}

The commercial software, COMSOL Multiphysics, was used to solve the 
numerical model. The computational domain was discretized with tetrahedral meshes. Grid independence check has been carried out to assure the accuracy of solutions. The total number of meshes is 956017 and the average mesh size is $5.48 \times 10^{-7} \mathrm{~mm}^{3}$. Direct solution procedure was conducted by using the Multifrontal Massively Parallel Solver (MUMPS). Key input parameters for the model are listed in Table 1. In order to fit the forward and reverse rate constants for $\mathrm{La}^{3+}$ and $\mathrm{Eu}^{3+}$, i.e. $k$ in Eq. 9, parametric analyses of rate constants were carried out based on the numerical model. Series of extraction curves of $\mathrm{La}^{3+}$ and $\mathrm{Eu}^{3+}$ were obtained at various rate constants. Least-square method was used to find the fitted values by comparing experimental data with numerical results.

\section{Results and discussion}

\subsection{Model validation}

The geometric dimensions of the droplet $(126 \mu \mathrm{m}$ in diameter and $250 \mu \mathrm{m}$ in length) and extraction conditions of the simulation model was kept the same as that of the microfluidic extraction experiments conducted by Nichols et al.[18]. The computational and experimental concentration of both $\mathrm{La}^{3+}$ and $\mathrm{Eu}^{3+}$ in aqueous solution along with extraction time was plotted in Fig. 2. From the results, a good agreement between modeling and experimental data indicates the validity of the presented model.

In order to obtain higher processing flux of lanthanide ions, a model with microchannel dimension of $1 \mathrm{~mm}$ in diameter was adopted in the following modeling 
analyses, and the organic to aqueous ratio was kept at 1:1.

\subsection{Extraction performance and limiting factors}

Fig. 3 depicts the concentration profiles of metal ions in aqueous solution against the mixing time on various flow velocities. As shown in the figure, extraction processes for both $\mathrm{La}^{3+}$ and $\mathrm{Eu}^{3+}$ were accelerated by increasing flow velocity. Fig. 4 shows the velocity and concentration distribution of lanthanides at different time in the microchannel. From the figure, we can find two main vortexes in both phases, which were also observed in previous experiments[29]. The existence of these vortexes can extremely boost the interphase mass transfer through replenishing reactants and removing products on interface[30, 31]. Since the extraction reaction of lanthanides only occurs at the interfacial surface, the extraction process of lanthanide from aqueous solution to organic solvent consists of three mass transfer steps in sequence, i.e., metal ions transfer from aqueous droplet to the interfacial surface, extraction reaction across the interface and mass transfer from the interface to solvent phase. As shown in Fig. 4, at the region near the interface, the concentration of ions drops while the one of complex rises at first. Because of the vortex flow, the concentration at central region decrease forming a butterfly shaped concentration distribution. Afterwards, ions transport from two circulation regions to the interface due to the convection and diffusion mass transfer. In contrast, the transport of lanthanide complex in organics solvent undergoes a reverse process.

From Fig. 3 and Fig. 4, it is found that much less time is spent for $\mathrm{La}^{3+}$ to 
achieve extraction equilibrium comparing with $\mathrm{Eu}^{3+}$ under the same hydrodynamic condition, which suggests that the extraction kinetics of $\mathrm{La}^{3+}$ is much faster than $\mathrm{Eu}^{3+}$. Damköhler number $(\mathrm{Da})$ is a valid index for evaluating the rate determining step in chemical engineering, which is defined as the ratio of the maximum extraction reaction rate to the rate of reactant transport to the interface[32]:

$$
D a=\frac{k c_{a q}^{n-1}}{k_{m}}=\frac{k c_{a q}^{n-1}}{\bar{D} / A+u / L_{s}}
$$

where $k$ is the reaction rate constant for extraction which can be expressed as $k_{f} k_{r}\left(\mathcal{c}_{\text {org }} / c_{a q)}\right.$, the length of slug $L_{s}$ is $1.5 \mathrm{~mm}$. For $\mathrm{Da}>1$, the reaction rate is faster than the rate of reactant transport to the interface. As a result, the overall rate of reaction is limited by mass transfer rate, which means the rate determining step is mass transport. On the other hand, $\mathrm{Da}<1$ means the reaction kinetics determine the overall reaction rate. Fig. 5 shows the relation between $\mathrm{Da}$ and flow velocity. It is found that the $\mathrm{Da}$ values of $\mathrm{La}^{3+}$ and $\mathrm{Eu}^{3+}$ extraction process are both much greater than one at low flow velocity, i.e. $0.1 \mathrm{~mm} \mathrm{~s}^{-1}$, which confirms that the performance limiting factors for both $\mathrm{La}^{3+}$ and $\mathrm{Eu}^{3+}$ extraction are inner-phase mass transfer. With flow velocity rising, $\mathrm{Da}$ values drop remarkably for both $\mathrm{La}^{3+}$ and $\mathrm{Eu}^{3+}$, which represents the mass transfer of inner-phase is enhanced. At a higher flow velocity, i.e. $10 \mathrm{~mm} \mathrm{~s}^{-1}$, Da values of $\mathrm{La}^{3+}$ and $\mathrm{Eu}^{3+}$ are around $10^{2}$ and 2.72 , respectively. The results imply that the extraction performance of $\mathrm{La}^{3+}$ is still limited by inner-phase mass transfer while the extraction of $\mathrm{Eu}^{3+}$ is determined by dual limiting factors, i.e. inner-phase mass transfer and extraction kinetics.

In order to identify the enhancement effect of flow velocity, here we define an 
intensification factor (Inf) as a ratio of the net generation rate of lanthanide complex on any flow velocity at mixing time of $10 \mathrm{~s}$ to the one on $0.1 \mathrm{~mm} \mathrm{~s}^{-1}$. This factor is an indication of intensification performance on extraction induced by flow velocity.

$$
\operatorname{Inf}=\left.\frac{R_{\text {gen }, u}}{R_{\text {gen }, 0.1}}\right|_{t=10 s}
$$

Fig. 6 plots the relation of intensification factor against flow velocity. From the results, the Inf of $\mathrm{Eu}^{3+}$ is found lower than $\mathrm{La}^{3+}$ for the whole range of flow velocity from 0.1 to $10 \mathrm{~mm} \mathrm{~s}^{-1}$. For extraction of $\mathrm{La}^{3+}$, the Inf increases dramatically by 2.4 at $2 \mathrm{~mm} \mathrm{~s}^{-1}$. It is because the rate determining step of $\mathrm{La}^{3+}$ extraction is inner-phase mass transfer which can be significantly accelerated by rising in flow velocity. Whereas, Inf grows slowly from 2 to $10 \mathrm{~mm} \mathrm{~s}^{-1}$. As shown in Fig. 3(a), the concentration of $\mathrm{La}^{3+}$ at $10 \mathrm{~s}$ drops rapidly from 2 to $10 \mathrm{~mm} \mathrm{~s}^{-1}$, which causes decline in generation rate of La complex. As a consequence, the increasing rate of Inf keeps slight at high flow velocity region. As for $\mathrm{Eu}^{3+}$, Inf rises considerably by 1.6 at $2 \mathrm{~mm} \mathrm{~s}^{-1}$ because of enhanced inner-phase mass transfer. As shown in Fig.5, the Da for Eu extraction drops by 2.72 at $10 \mathrm{~mm} \mathrm{~s}^{-1}$. As a matter of fact, with the rising of flow velocity, the effect of reaction kinetics on $\mathrm{Eu}^{3+}$ extraction rate begins to emerge, which result in the fall of increasing rate of Inf.

\subsection{Mechanism of separation intensification}

For a given microchannel configuration, the mixing length is fixed. In this work, a microchannel of $100 \mathrm{~mm}$ in length was studied. Increase in flow velocity will result in linear decline in residence time of mixing. Fig. 7 shows the concentration profiles 
of metal ions in aqueous solution along the mixing distance on various flow velocities. Although extraction process can be accelerated through rising flow velocity as indicated in Fig. 3, there is not enough residence time in the microchannel to achieve extraction equilibrium at high flow velocities for microchannel with constant length. The extraction percentage $(E)$ of lanthanide at the outlet of the microchannel is given as follow:

$$
E=\frac{c_{o r g, i} V_{o r g}}{c_{o r g, i} V_{o r g}+c_{a q, i} V_{a q}}
$$

As shown in the Fig. 8, the extraction percentage drops sharply with increasing in flow velocity beyond $0.1 \mathrm{~mm} \mathrm{~s}^{-1}$. Therefore, there is a trade-off between extraction kinetics and extraction percentage. Graetz number $(\mathrm{Gz})$ was utilized to access the ability of achieving high extraction percentage for microfluidic extraction. In general, $\mathrm{Gz}$ is defined as the ratio of time scale of diffusion towards reactive surface to that of convection in the flow direction[33]. However, for microfluidic extraction based on droplet configuration, the reactants transport to the interface relies on not only diffusion, but also inner-phase convection. Therefore, a modified $\mathrm{Gz}$ is defined here as the ratio of time scale of total mass transport towards interface to that of convection transport in the flow direction:

$$
G z=\frac{\overline{t_{m}}}{\overline{t_{c}}}=\frac{A / \bar{D}+L_{s} / u}{L / u}
$$

For the case of $\mathrm{Gz}>1$, i.e. total mass transport time scale is longer than the residence time scale. In this circumstance, reactants do not have enough time to take part in the extraction reaction before exiting the microchannel, resulting in low extraction 
percentage. On the other hand, for $\mathrm{Gz}<1$, there is enough time for reactants to cross the interface, in which case high extraction percentage can be achieved. As shown in Fig. 9, Gz value reduces from 2.38 to 0.039 with flow velocity decreased from $10 \mathrm{~mm}$ $\mathrm{s}^{-1}$ to $0.1 \mathrm{~mm} \mathrm{~s}^{-1}$, while extraction percentage of $\mathrm{La}^{3+}$ and $\mathrm{Eu}^{3+}$ increases from $52 \%$ to $89 \%$ and from $23 \%$ to $84 \%$, respectively.

Separation performance of the microfluidic extraction system can be evaluated by separation factor which is given as follow[17]:

$$
\beta_{L a / E u}=\frac{D_{L a}}{D_{E u}}
$$

where $D_{L a}$ and $D_{E u}$ represents the distribution ratio of $\mathrm{La}^{3+}$ and $\mathrm{Eu}^{3+}$, respectively. Distribution ratio is defined as the concentration of lanthanide ligand complex to that of lanthanide ions:

$$
D_{i}=\frac{c_{o r g, i}}{c_{a q, i}}
$$

In conventional separation devices, such as mixer-settler, separation of species is achieved on equilibrium condition, corresponding to a separation factor of 1.60 for $\mathrm{La}^{3+} / \mathrm{Eu}^{3+}$ separation here. In microfluidic extraction, as shown in Fig. 8, with flow velocity increased from $0.01 \mathrm{~mm} \mathrm{~s}^{-1}$ to $1 \mathrm{~mm} \mathrm{~s}^{-1}$, the separation factor boosts from 1.60 to 2.54 , and then reaches a short plateau as flow velocity rises to $2 \mathrm{~mm} \mathrm{~s}^{-1}$. With further increase in flow velocity, as high as 3.53 of separation factor can be achieved at a flow velocity of $10 \mathrm{~mm} \mathrm{~s}^{-1}$. Similar effect of flow velocity on separation performance in a droplet-based microfluidic extraction system was observed in previous experiments[17]. Two factors contribute to the separation enhancement of 
microfluidic extraction as compared with conventional devices. First, the distinction of extraction kinetic rate plays a key role in separation intensification. Since extraction kinetics of $\mathrm{La}^{3+}$ is much faster than $\mathrm{Eu}^{3+}$, which is confirmed in Fig. 2 and Fig. 3, separation enhancement can be achieved through controlling the residence time of lanthanides in the microchannel. Second, intensification effect of flow velocity for $\mathrm{La}^{3+}$ and $\mathrm{Eu}^{3+}$ extraction is distinguished, as shown in Fig. 5. Rising of flow velocity will further expand the difference of extraction kinetics between $\mathrm{La}^{3+}$ and $\mathrm{Eu}^{3+}$. The separation performance can be manipulated through controlling flow velocity.

\section{Conclusion}

In this paper, numerical analyses were carried out to understand the characteristics of lanthanide extraction in a droplet-based microfluidic extraction system. Two kinds of lanthanide ions with distinguished extraction kinetics, i.e., $\mathrm{La}^{3+}$ and $\mathrm{Eu}^{3+}$, were studied. Inner-phase mass transfer was identified as the extraction performance limiting factors for $\mathrm{La}^{3+}$ for the range of the flow velocities studied. In contrast, for $\mathrm{Eu}^{3+}$, the performance determining step changes from inner-phase mass transfer to a dual-limiting mechanism as flow velocity increases. Microfluidic extraction is verified a promising configuration for efficient separation of lanthanides. As high as 3.53 of separation factor for $\mathrm{La}^{3+} / \mathrm{Eu}^{3+}$ can be achieved at a flow velocity of $10 \mathrm{~mm} \mathrm{~s}^{-1}$. This study provides a mechanistic understanding of extraction and separation of lanthanide in droplet-based microfluidic extraction technique. 


\section{Acknowledgments}

The research work presented in this paper is supported by Natural Science Foundation of Jiangxi Province, China (20171BAB216023), Research Project of Jiangxi Department of Education, China (GJJ150639), Program for Excellent Young Talents, JXUST. 


\section{Reference}

[1] J.-C.G. Bünzli, Benefiting from the Unique Properties of Lanthanide Ions, Accounts of Chemical Research 39 (2006) 53-61.

[2] F. Wang, Y. Han, C.S. Lim, Y. Lu, J. Wang, J. Xu, H. Chen, C. Zhang, M. Hong, X. Liu, Simultaneous phase and size control of upconversion nanocrystals through lanthanide doping, Nature 463 (2010) 1061.

[3] S. Ye, Q. Tang, Y. Wang, W. Fei, PIV measurement and CFD simulation of liquid-liquid flow of a settler in rare earths solvent extraction mixer-settler, International Journal of Heat and Fluid Flow 62 (2016) 568-576.

[4] D. Ciceri, J.M. Perera, G.W. Stevens, The use of microfluidic devices in solvent extraction, Journal of Chemical Technology \& Biotechnology 89 (2014) 771-786.

[5] L. Shui, J.C.T. Eijkel, A. van den Berg, Multiphase flow in microfluidic systems Control and applications of droplets and interfaces, Advances in Colloid and Interface Science 133 (2007) 35-49.

[6] A. Sahu, A.B. Vir, L.N.S. Molleti, S. Ramji, S. Pushpavanam, Comparison of liquid-liquid extraction in batch systems and micro-channels, Chemical Engineering and Processing: Process Intensification 104 (2016) 190-200.

[7] O. Tamagawa, A. Muto, Development of cesium ion extraction process using a slug flow microreactor, Chemical Engineering Journal 167 (2011) 700-704.

[8] K.K. Singh, A.U. Renjith, K.T. Shenoy, Liquid-liquid extraction in microchannels and conventional stage-wise extractors: A comparative study, Chemical Engineering and Processing: Process Intensification 98 (2015) 95-105.

[9] D. Ciceri, L.R. Mason, D.J.E. Harvie, J.M. Perera, G.W. Stevens, Extraction kinetics of $\mathrm{Fe}(\mathrm{III})$ by di-(2-ethylhexyl) phosphoric acid using a Y-Y shaped microfluidic device, Chemical Engineering Research and Design 92 (2014) 571-580. [10] A. Aota, M. Nonaka, A. Hibara, T. Kitamori, Countercurrent Laminar Microflow for Highly Efficient Solvent Extraction, Angewandte Chemie 119 (2007) 896-898. [11] M. N. Kashid, A. Renken, L. Kiwi-Minsker, Influence of Flow Regime on Mass Transfer in Different Types of Microchannels, Industrial \& Engineering Chemistry 
Research 50 (2011) 6906-6914.

[12] J.H. Xu, J. Tan, S.W. Li, G.S. Luo, Enhancement of mass transfer performance of liquid-liquid system by droplet flow in microchannels, Chemical Engineering Journal 141 (2008) 242-249.

[13] X. Wang, G. Liu, K. Wang, G. Luo, Measurement of internal flow field during droplet formation process accompanied with mass transfer, Microfluidics and Nanofluidics 19 (2015) 757-766.

[14] S. Ma, J.M. Sherwood, W.T.S. Huck, S. Balabani, On the flow topology inside droplets moving in rectangular microchannels, Lab on a Chip 14 (2014) 3611-3620.

[15] A.-L. Dessimoz, L. Cavin, A. Renken, L. Kiwi-Minsker, Liquid-liquid two-phase flow patterns and mass transfer characteristics in rectangular glass microreactors, Chemical Engineering Science 63 (2008) 4035-4044.

[16] S. Nishihama, Y. Tajiri, K. Yoshizuka, Separation of lanthanides using micro solvent extraction system, Ars Separatoria Acta (2006) 18-26.

[17] L.-h. Zhang, J.-h. Peng, S.-h. Ju, L.-b. Zhang, L.-q. Dai, N.-s. Liu, Microfluidic solvent extraction and separation of cobalt and nickel, RSC Advances 4 (2014) 16081-16086.

[18] K.P. Nichols, R.R. Pompano, L. Li, A.V. Gelis, R.F. Ismagilov, Toward Mechanistic Understanding of Nuclear Reprocessing Chemistries by Quantifying Lanthanide Solvent Extraction Kinetics via Microfluidics with Constant Interfacial Area and Rapid Mixing, Journal of the American Chemical Society 133 (2011) 15721-15729.

[19] M. Kubicek, A.H. Bork, J.L.M. Rupp, Perovskite oxides - a review on a versatile material class for solar-to-fuel conversion processes, Journal of Materials Chemistry A 5 (2017) 11983-12000.

[20] H. Zhu, P. Zhang, S. Dai, Recent Advances of Lanthanum-Based Perovskite Oxides for Catalysis, ACS Catalysis 5 (2015) 6370-6385.

[21] Z.M. Geballe, H. Liu, A.K. Mishra, M. Ahart, M. Somayazulu, Y. Meng, M. Baldini, R.J. Hemley, Synthesis and Stability of Lanthanum Superhydrides, Angewandte Chemie International Edition 57 (2018) 688-692. 
[22] P. Lacorre, F. Goutenoire, O. Bohnke, R. Retoux, Y. Laligant, Designing fast oxide-ion conductors based on La2Mo2O9, Nature 404 (2000) 856.

[23] A.J. Jacobson, Materials for Solid Oxide Fuel Cells, Chemistry of Materials 22 (2010) 660-674.

[24] H. Terraschke, C. Wickleder, UV, Blue, Green, Yellow, Red, and Small: Newest Developments on Eu2+-Doped Nanophosphors, Chemical reviews 115 (2015) 11352-11378.

[25] C. Bouzigues, T. Gacoin, A. Alexandrou, Biological Applications of Rare-Earth Based Nanoparticles, ACS Nano 5 (2011) 8488-8505.

[26] M. Lubej, U. Novak, M. Liu, M. Martelanc, M. Franko, I. Plazl, Microfluidic droplet-based liquid-liquid extraction: online model validation, Lab on a Chip 15 (2015) 2233-2239.

[27] N. Harries, J.R. Burns, D.A. Barrow, C. Ramshaw, A numerical model for segmented flow in a microreactor, International Journal of Heat and Mass Transfer 46 (2003) 3313-3322.

[28] S.K.R. Cherlo, S. Kariveti, S. Pushpavanam, Experimental and Numerical Investigations of Two-Phase (Liquid-Liquid) Flow Behavior in Rectangular Microchannels, Industrial \& Engineering Chemistry Research 49 (2010) 893-899.

[29] P. Mary, V. Studer, P. Tabeling, Microfluidic Droplet-Based Liquid-Liquid Extraction, Analytical Chemistry 80 (2008) 2680-2687.

[30] J.R. Burns, C. Ramshaw, The intensification of rapid reactions in multiphase systems using slug flow in capillaries, Lab on a Chip 1 (2001) 10-15.

[31] H. Shao, Y. LÜ, K. Wang, G. Luo, An Experimental Study of Liquid-Liquid Microflow Pattern Maps Accompanied with Mass Transfer, Chinese Journal of Chemical Engineering 20 (2012) 18-26.

[32] J. Xuan, H.Z. Wang, D.Y.C. Leung, M.K.H. Leung, H. Xu, L. Zhang, Y. Shen, Theoretical Graetz-Damkohler modeling of an air-breathing microfluidic fuel cell, Journal of Power Sources 231 (2013) 1-5.

[33] H. Xu, H. Zhang, H. Wang, D.Y.C. Leung, L. Zhang, J. Cao, K. Jiao, J. Xuan, Counter-flow formic acid microfluidic fuel cell with high fuel utilization exceeding 
90\%, Applied Energy 160 (2015) 930-936. 


\section{List of tables}

Table 1. Key input parameters for the numerical model. 
Table 1. Key input parameters for the numerical model.

\begin{tabular}{llll}
\hline Parameters & Unit & Value & Refs. \\
\hline$\alpha$ & degree & 155 & {$[18]$} \\
$\mu_{a}$ & mPa s & 1 & {$[18]$} \\
$\mu_{o}$ & $\mathrm{mPa} \mathrm{s}$ & 1.383 & {$[18]$} \\
$k_{f, L a}$ & 48 & fitted \\
$k_{r, L a}$ & $\mathrm{~s}^{-1}$ & 3 & fitted \\
$k_{f, E u}$ & $\mathrm{~s}^{-1}$ & 1.05 & fitted \\
$k_{r, E u}$ & $\mathrm{~s}^{-1}$ & & fitted \\
$\bar{D}$ & $\mathrm{~s}^{-1}$ & 0.15 & [18] \\
\hline
\end{tabular}




\section{List of figures}

Fig. 1. The geometry of computational domain

Fig. 2. Experimental and numerical results of lanthanide's concentration in droplet for microfluidic extraction of $\mathrm{La}^{3+}$ and $\mathrm{Eu}^{3+}$

Fig. 3. Simulated concentration profiles of (a) $\mathrm{La}^{3+}$ and (b) $\mathrm{Eu}^{3+}$ in droplet against mixing time at various flow velocities.

Fig. 4. Velocity and concentration distributions of lanthanides at $10 \mathrm{~s}, 50 \mathrm{~s}, 100 \mathrm{~s}$ in microchannel on flow velocity of $1 \mathrm{~mm} \mathrm{~s}^{-1}$.

Fig. 5. Da values of $\mathrm{La}^{3+}$ and $\mathrm{Eu}^{3+}$ against flow velocity

Fig. 6. Intensification factor of $\mathrm{La}^{3+}$ and $\mathrm{Eu}^{3+}$ against flow velocity

Fig. 7. Concentration profiles of (a) $\mathrm{La}^{3+}$ and (b) $\mathrm{Eu}^{3+}$ in droplet under various flow velocities along with mixing distance

Fig. 8. Extraction percentage of $\mathrm{La}^{3+}$ and $\mathrm{Eu}^{3+}$, separation factor against flow velocity Fig. 9. Gz value against flow velocity 
Fig. 1. The geometry of computational domain

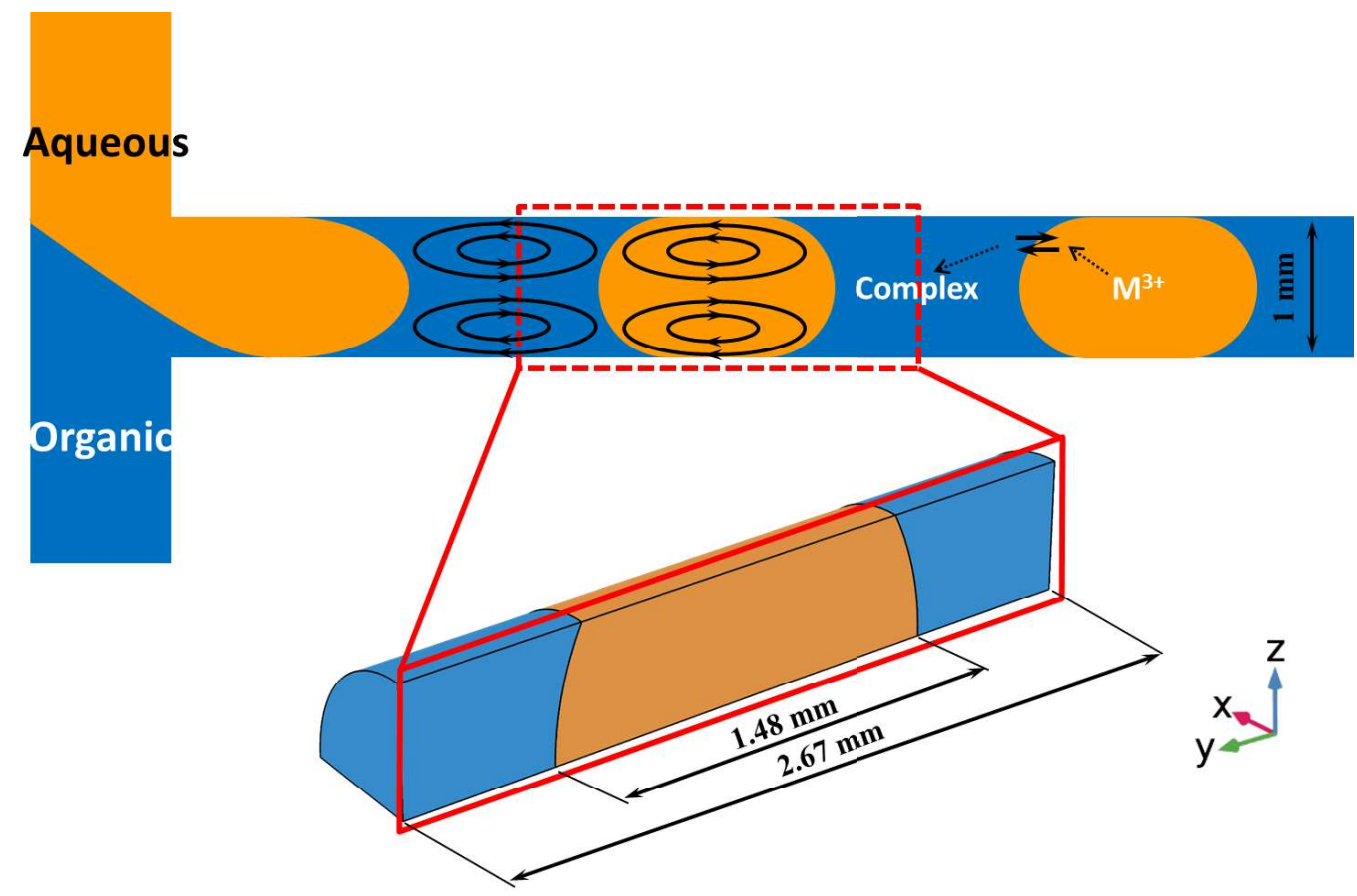


Fig. 2. Experimental and numerical results of lanthanide's concentration in droplet for microfluidic extraction of $\mathrm{La}^{3+}$ and $\mathrm{Eu}^{3+}$

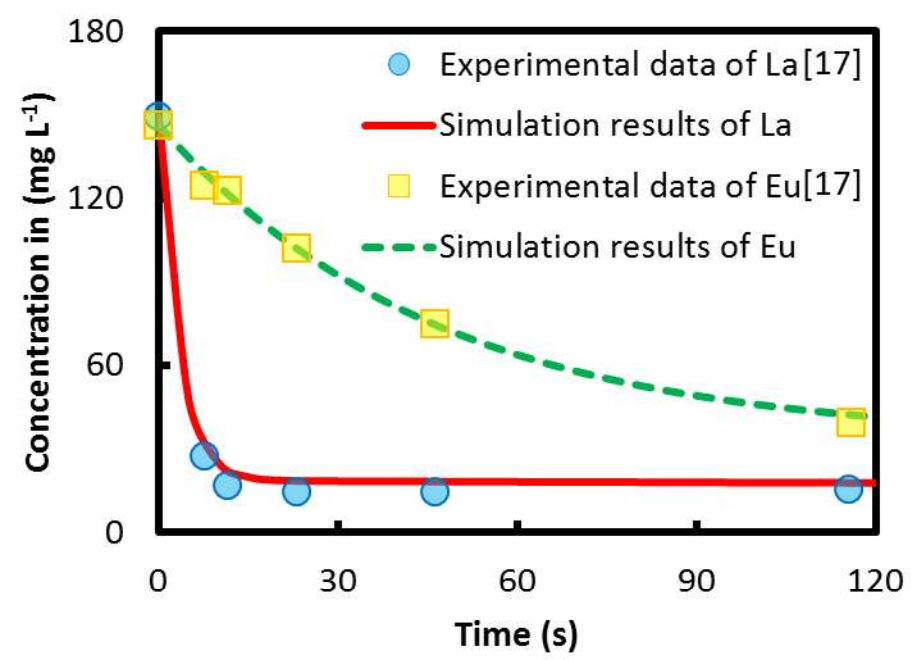


Fig. 3. Simulated concentration profiles of (a) $\mathrm{La}^{3+}$ and (b) $\mathrm{Eu}^{3+}$ in droplet against mixing time at various flow velocities.
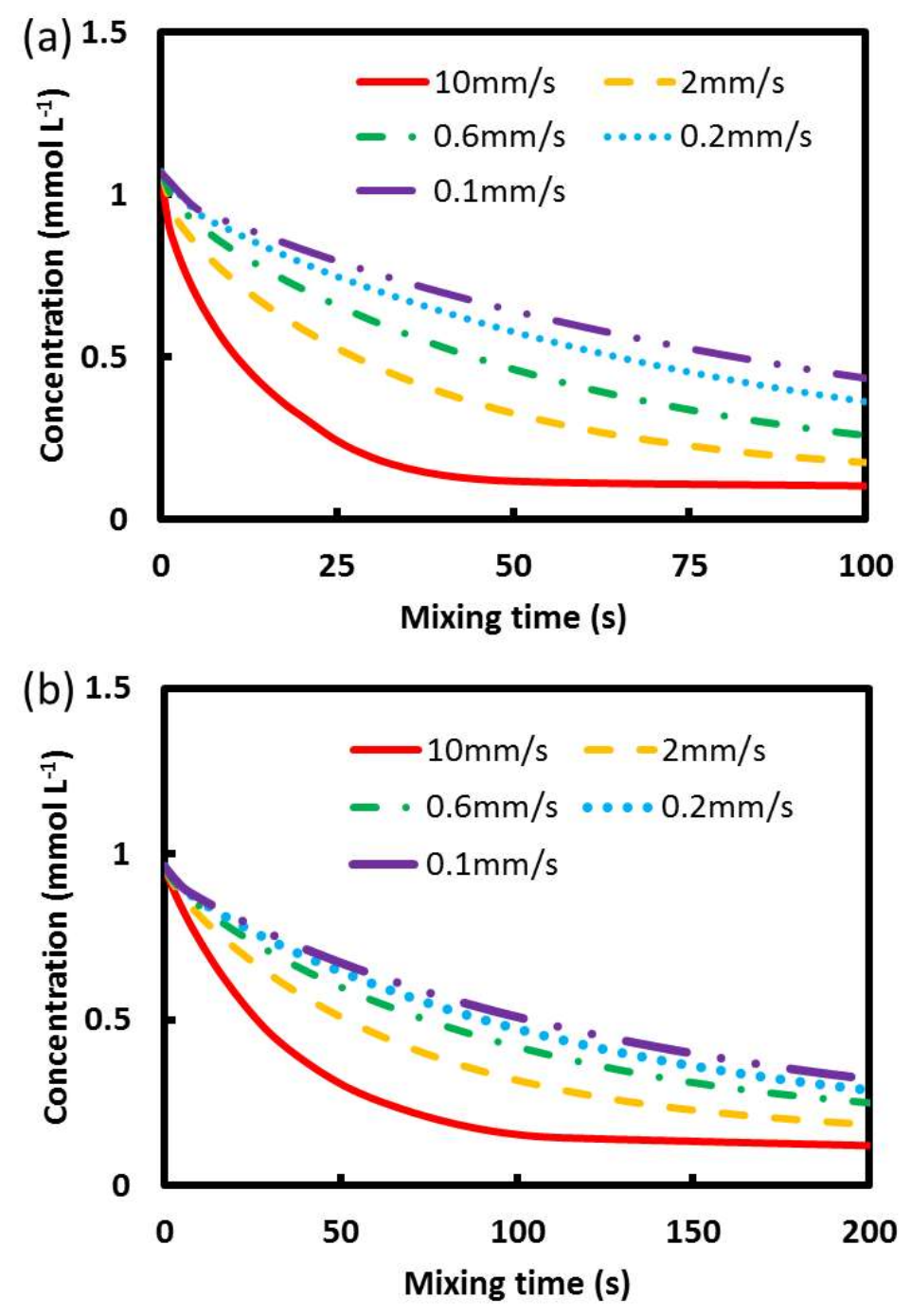
Fig. 4. Velocity and concentration distributions of lanthanides at $10 \mathrm{~s}, 50 \mathrm{~s}, 100 \mathrm{~s}$ in microchannel on flow velocity of $1 \mathrm{~mm} \mathrm{~s}^{-1}$.

La

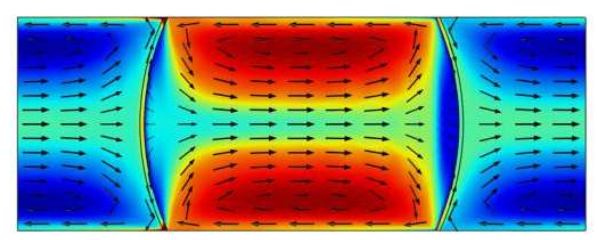

50 s

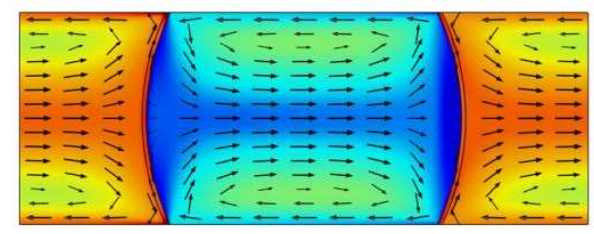

100s

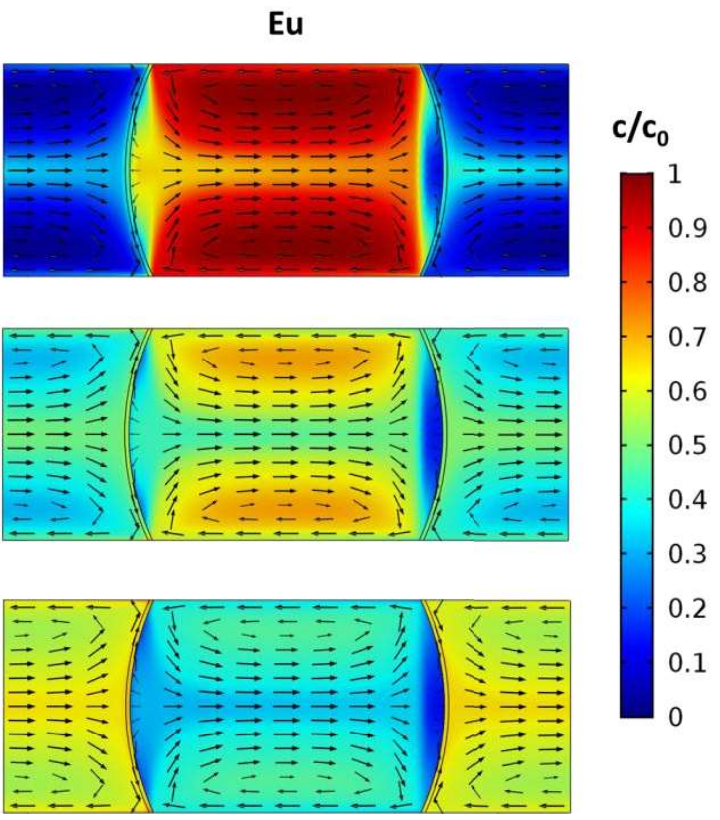


Fig. 5. Da values of $\mathrm{La}^{3+}$ and $\mathrm{Eu}^{3+}$ against flow velocity

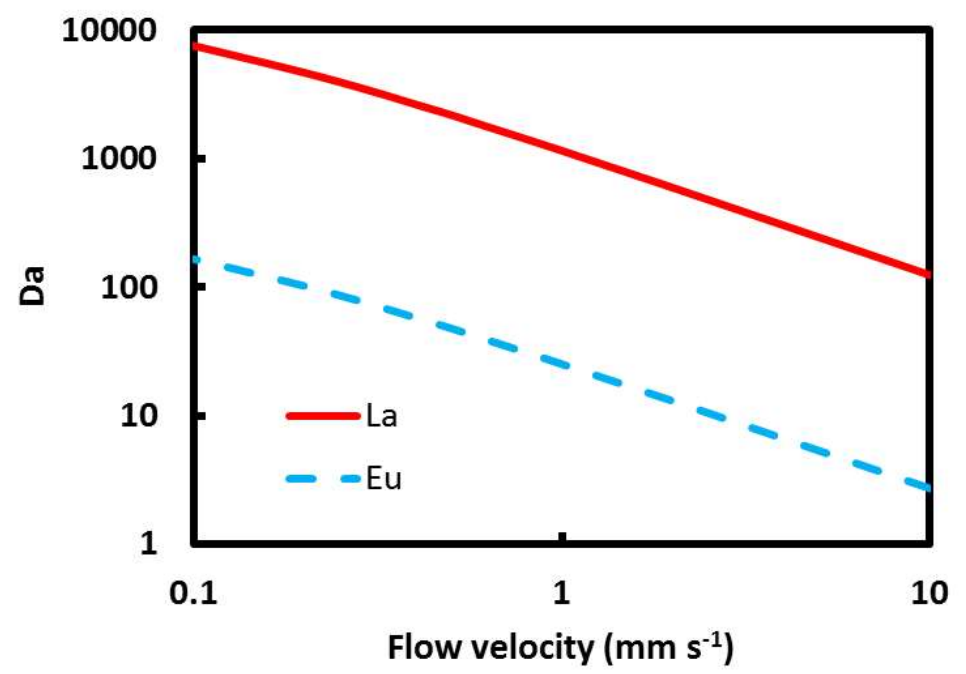


Fig. 6. Intensification factor of $\mathrm{La}^{3+}$ and $\mathrm{Eu}^{3+}$ against flow velocity

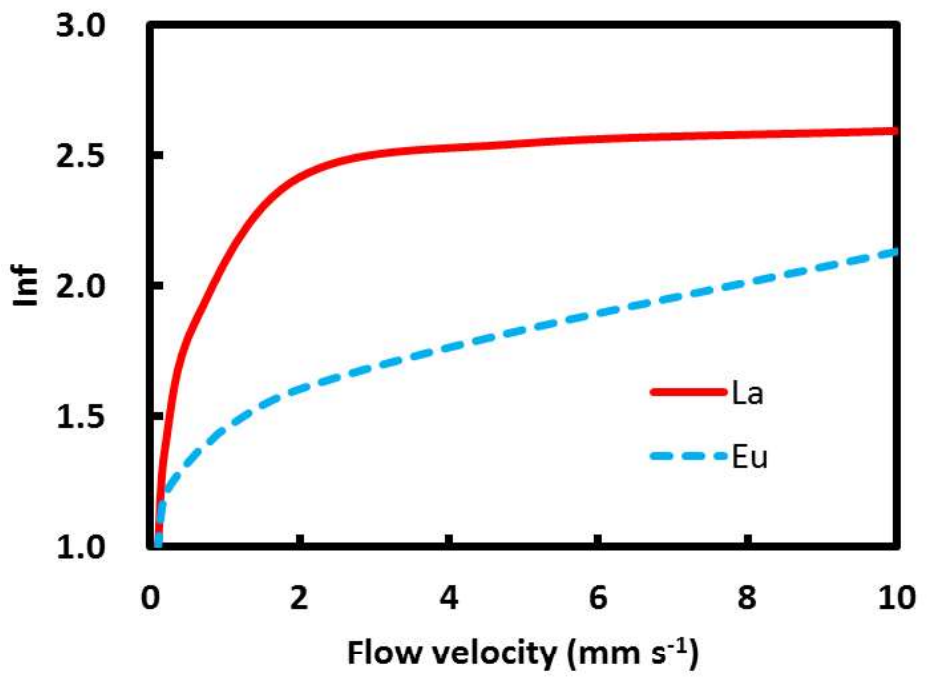


Fig. 7. Concentration profiles of (a) $\mathrm{La}^{3+}$ and (b) $\mathrm{Eu}^{3+}$ in droplet under various flow velocities along with mixing distance
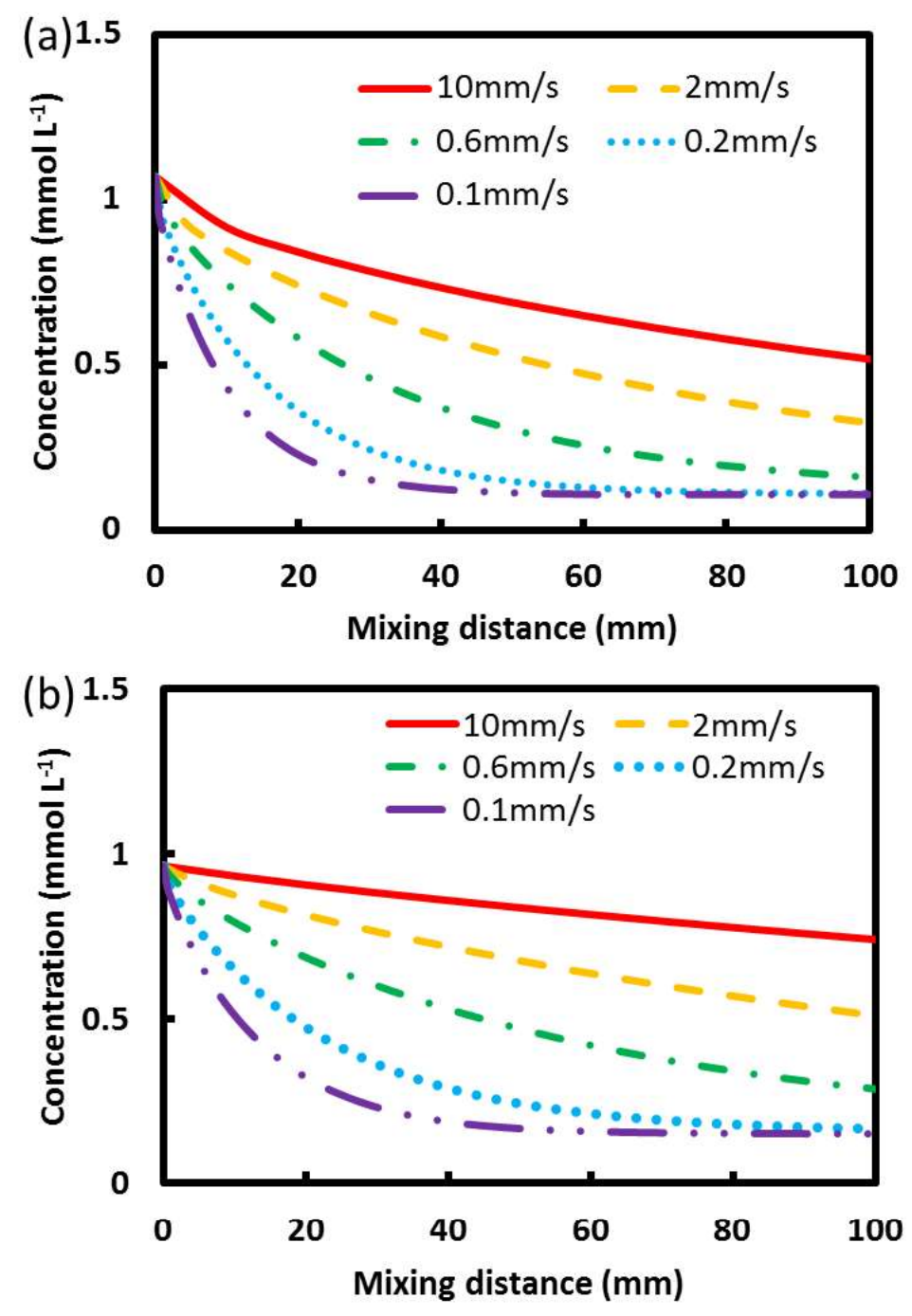
Fig. 8. Extraction percentage of $\mathrm{La}^{3+}$ and $\mathrm{Eu}^{3+}$, separation factor against flow velocity

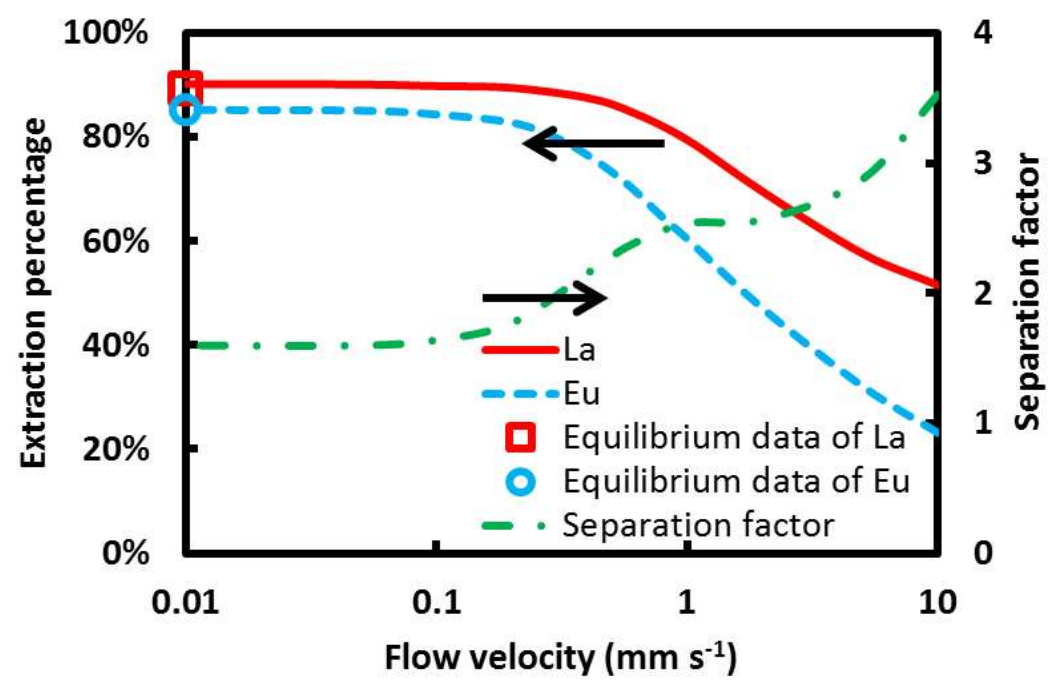


Fig. 9. Gz value against flow velocity

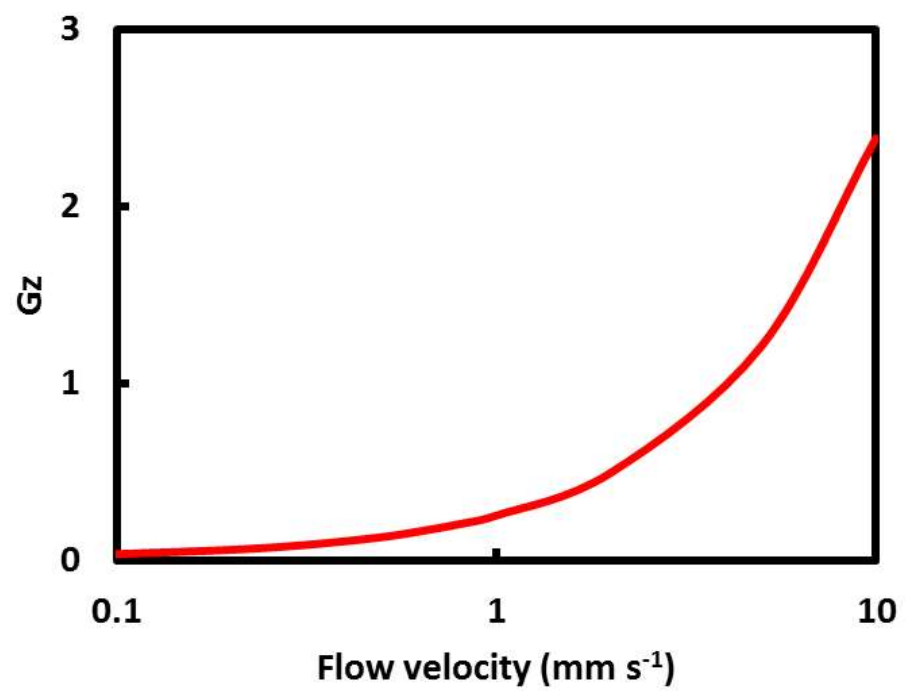

